\title{
Macrophages and fibroblasts underpin skin immune responses
}

\author{
Makoto Sugaya* \\ Department of Dermatology, Faculty of Medicine, International University of Health and Welfare, Narita, Chiba Prefecture \\ 286-8520, Japan
}

*Correspondence: Makoto Sugaya, Department of Dermatology, Faculty of Medicine, International University of Health and Welfare, Narita, Chiba Prefecture 286-8520, Japan. sugayamder@iuhw.ac.jp

Academic Editor: Dominique J. Charron, Hospital Saint Louis, France

Received: May 30, 2021 Accepted: August 19, 2021 Published: August 31, 2021

Cite this article: Sugaya M. Macrophages and fibroblasts underpin skin immune responses. Explor Immunol. 2021;1:226-42. https://doi.org/10.37349/ei.2021.00015

\begin{abstract}
There are various types of skin immune responses including inflammatory skin diseases and skin malignancy. Macrophages and fibroblasts are skin resident cells that had been overlooked in terms of immunological research targets. In this review, cross talk among macrophages, fibroblasts, and migratory immune cells in skin diseases such as atopic dermatitis (AD), contact hypersensitivity, psoriasis, systemic sclerosis, melanoma, and cutaneous T-cell lymphoma is described. Macrophages are important in AD by antigen-presenting phagocytosis, production of inflammatory cytokines, removal of apoptotic cells, and mediating clusters between dendritic cells (DCs) and T cells. They are also increased in lesional skin of psoriasis, especially in stable plaques, and an increased ratio of M1/M2 macrophages and tumor necrosis factor- $\alpha$ production by macrophages are essential for development of psoriasis. The progression of skin malignancy is mediated by macrophages through promotion of tumor survival pathways via expression of cytokines and growth factors, interaction with regulatory T cells (Tregs) and myeloid-derived suppressor cells, and suppression of function of tumor-infiltrating $\mathrm{T}$ cells by immunosuppressive cytokines and programmed death-ligand (PD-L)1. Fibroblasts play important roles in development and maintenance of AD lesions through expression of CC chemokine ligand (CCL)17, CCL11, CCL26, C-X-C motif chemokine ligand (CXCL)12, CCL19, and periostin, interacting with T helper (Th)2 cells, natural killer T (NKT) cells, DCs, and keratinocytes. They also play important roles in psoriasis, expressing interleukin (IL)-8 and vascular endothelial growth factor, production of fibronectin, and changes in the proteomic profiles. Fibroblasts have a critical role in the progression skin malignancy via expression of cytokines, suppression natural killer (NK) functions, and establishment of Th2-dominant microenvironment. Thus, cross talk among macrophages, fibroblasts, and migratory immune cells including T cells, DCs, and NK cells in skin diseases is important and those skin-resident cells are attracting therapeutic targets in the near future.
\end{abstract}

\section{Keywords}

Macrophages, fibroblasts, atopic dermatitis, contact hypersensitivity, psoriasis, systemic sclerosis, melanoma, cutaneous T-cell lymphoma 


\section{Introduction}

There are various types of skin immune responses including inflammatory skin diseases and skin malignancy. The main player in immune responses is T cells that can migrate from blood into the skin via soluble factors such as chemokines. Some T cells can stay in the skin for a long time as resident memory $\mathrm{T}$ cells, playing important roles in the pathogenesis of skin diseases with clear borderlines such as psoriasis $[1,2]$. In case of skin malignancy, tumor-infiltrating T cells (TILs) are essential, which can be one of prognostic indicators. In these days, activating TILs by checkpoint inhibitors is a promising treatment option for skin tumors such as melanoma and Merkel cell carcinoma [3-5]. In the lesional skin of either inflammatory diseases or skin malignancy, however, there are varieties of cells other than $\mathrm{T}$ cells mediating skin immune responses.

Macrophages and fibroblasts are skin resident cells that had been overlooked in terms of immunological research targets. Their progenitors migrate into various tissues including skin and have distinct appearances and cell surface phenotypes. In this review, the author would like to focus on cross talk among macrophages, fibroblasts, and migratory immune cells such as T cells and dendritic cells (DCs) in skin diseases.

\section{Role of macrophages in atopic dermatitis and contact hypersensitivity}

Tissue-resident macrophage populations are heterogenous, showing substantial tropism relative to their microenvironment. They are important for tissue homeostasis and tissue remodeling. It is now clear that macrophages subpopulations can be categorized by expression of different types of receptor and cytokine/chemokine [6-11]. Classically activated macrophages, also called M1 cells, are induced by interferon (IFN) $\gamma$ and have an ability to present antigens to T cells. They produce inflammatory cytokines such as interleukin (IL)-1 $\beta$, tumor necrosis factor (TNF)- $\alpha$, IL-6, IL-12, and IL-23, involved in tissue damage, inflammation, and tumor inhibition. In contrast, alternatively activated macrophages, also called M2 cells, are induced by IL- 4 , which promote type 2 responses. They produce IL-10 and transforming growth factor (TGF)- $\beta$, and show high expression of several receptors such as class A scavenger receptor, mannose receptor, dectin-1, CD209, and CD163. They are involved in tissue repair, tumor cell growth, and angiogenesis. In the setting of inflammation, macrophages play essential roles such as antigen presentation, phagocytosis, and immunomodulation through production of cytokines and growth factors.

Atopic dermatitis (AD) is characterized by relapsing eczema with pruritus. Most patients with AD have family history of allergic diseases or past history of asthma, rhinitis, or conjunctivitis [12]. Although the skin disease may be cleared by adolescence in most cases, it often becomes a chronic condition. AD has significant negative social and economic impacts, decreasing the quality of life of the patients and their families. Skin conditions of $\mathrm{AD}$ children influence quality of life of both children and their parents $[13,14]$. Drug therapy with topical steroid or topical tacrolimus, skin care with moisturizers for dry skin, and investigation and elimination of exacerbating factors are three major parts of the treatment [12]. The pathogenesis of $\mathrm{AD}$ can be explained from the perspectives of the skin barrier, allergic inflammation, and severe pruritus [15]. With regards to allergic immune responses, cytokines expressed by T helper (Th) 2 cells and group 2 innate lymphoid cells such as IL-4, IL-5, and IL-13 have been regarded as main players of AD [16-18].

Although T cells are the key player in AD, macrophages and cytokines expressed by them exaggerate skin inflammation in AD. Macrophages are known to accumulate in acutely and chronically inflamed AD skin [19]. During the early phase, macrophages exert proinflammatory functions like antigen-presenting phagocytosis and production of inflammatory cytokines. In the late phase, many immune cells go into apoptosis and need to be removed efficiently. Increased macrophages may be associated with removal of apoptotic cells. IL-33, a member of the IL-1 family of cytokines activating various immune cells through its receptor ST2 [16, 17] is produced by macrophages as well as epithelial cells. The IL-33-induced production of pro-inflammatory cytokines play important roles, aggravating atopic diseases such as $\mathrm{AD}$ and asthma.

The importance of macrophages was also reported in contact hypersensitivity (CHS). For example, arginase-1 deficiency in lysozyme $\mathrm{M}$ (LysM) ${ }^{+}$cells including monocytes and macrophages resulted in exacerbation of CHS through upregulated inducible nitric oxide synthase (iNOS) expression [20]. Consistently, we recently reported that CHS in CX3C chemokine receptor 1 (CX3CR1)-deficient mice was attenuated with 
increased expression of arginase-1, which is a representative M2 macrophage marker [21]. Furthermore, dermal macrophage depletion in wild type (WT) mice before elicitation phase suppressed CHS, suggesting important roles of macrophages in CHS. Dermal macrophages are also important for the formation of clusters between DCs and effector T cells in dermal perivascular areas in CHS [22]. IL- $1 \alpha$-induced production of C-X-C motif chemokine ligand (CXCL) 2 by dermal macrophages was essential for DC clustering. Glucocorticoids are widely used in the treatment of allergic skin diseases such as CHS. CHS was suppressed by glucocorticoids only at elicitation phase in a mouse model. Inactivation of their receptor gene in keratinocytes or $\mathrm{T}$ cells did not attenuate the effects of glucocorticoids, whereas its ablation in macrophages and neutrophils lost the suppressive effect of glucocorticoids [23]. Taken together, macrophages are important in AD and CHS, especially at elicitation phase, by antigen-presenting phagocytosis, production of inflammatory cytokines, removal of apoptotic cells, and mediating clusters between DCs and T cells (Figure 1).

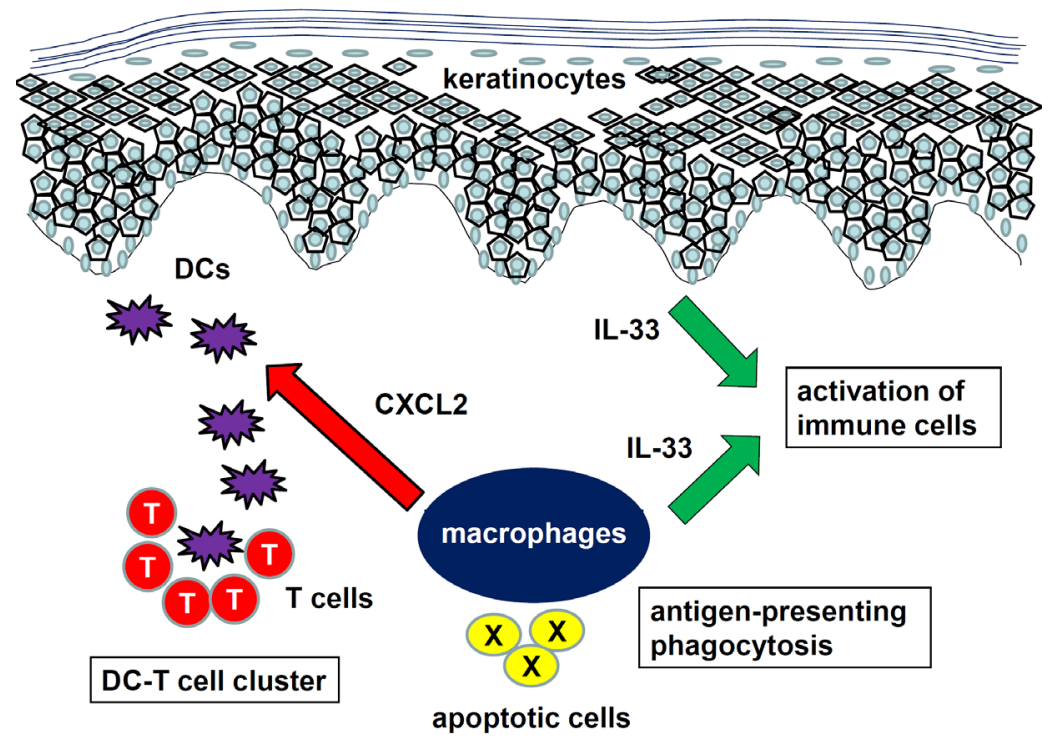

Figure 1. Role of macrophages in $\mathrm{AD}$ and $\mathrm{CHS}$. Macrophages mediate antigen-presenting phagocytosis, production of inflammatory cytokines such as IL-33, and cluster formation between DCs and T cells

\section{Role of macrophages in psoriasis}

Psoriasis is a chronic inflammatory skin disease that affects $2-3 \%$ of the worldwide population. It is mediated by cross talk between epidermal keratinocytes, dermal vascular cells, and immune cells, including DCs, macrophages, and T cells. The disease is characterized by epidermal hyperproliferation, leukocyte infiltration, and vascular proliferation in the papillary dermis. The disease is associated with several important medical conditions such as arthritis, depression, and cardiometabolic syndrome [24]. Biologics targeting IL-23, IL-17, and IL-17RA are approved for clinical use and show excellent efficacy [25].

The expression levels of monocyte and macrophage markers, such as CD14, CD33, CD68, and CD163 were increased in lesional tissues from psoriasis compared to normal tissues [26]. In the imiquimod (IMQ)induced psoriasis mouse model, dermal macrophages started to increase during the late phase, whereas the number of Langerhans cells increased continuously [27]. The early and late phases of IMQ-induced psoriaticlike inflammation correlated with the presence of distinct types of myeloid cells, with neutrophils, monocytes, and DCs dominating the early phase and Langerhans cells and macrophages transiently increasing during the late phase. This is consistent with psoriasis lesional skin, where neutrophils are increased at early phase and macrophages and cell types mediating adaptive immunity predominate in stable plaques.

The function of macrophages in psoriasis has been studied using mouse models. Depletion of macrophages by injection of clodronate-containing liposome significantly attenuated IMQ-induced psoriatic responses $[26,28]$. Expression of $M 1$ macrophage markers was higher than that of M2 macrophage markers in the psoriatic tissues. A shift from a lower M1/M2 macrophage ratio in the tissues from control mice to a higher M1/M2 macrophage ratio in the tissues from IMQ-treated mice was reported [26]. Consistently, we showed that IMQ-induced psoriasis-like dermatitis was attenuated in CX3CR1\% mice compared to WT mice 
with decrease in M1 macrophage infiltration and increase in CC chemokine receptor (CCR) $2^{+}$population in dermal macrophages [28]. Decreased macrophage number in the skin of naïve CX3CR1\% mice suggested the importance of CX3CR1 for migration of resident macrophages in mice. Interaction between CC chemokine ligand (CCL) 2 and CCR2 probably compensated for loss of CX3CR1, which was consistent with the fact that CX3CR1 and CCR2 have independent functions in recruiting macrophages [29]. The CD18 hypomorphic PL/J mice developed chronic skin inflammation clinically resembling human psoriasis. Activated macrophages, an important source of TNF- $\alpha$, were significantly increased in lesional skin as well as in inflamed skin draining lymph nodes of the affected mice [30]. Both depletion of macrophages and neutralization of TNF- $\alpha$ resulted in a significant reduction of psoriasiform skin inflammation, suggesting that psoriasiform skin inflammation is maintained by efficient recruitment and activation of macrophages. Another mouse psoriasis model was epidermis-specific deletion of inhibitor of nuclear factor-kappaB (NF-kappaB) kinase 2. In these mice, skin disease was significantly improved when the mice were treated with a TNF- $\alpha$-neutralizing agent [31]. Elimination of skin macrophages by subcutaneous injection of clodronate liposomes was accompanied by inhibition of granulocyte migration into the skin and resulted in a dramatic attenuation of psoriasis-like skin inflammation. Targeted deletion of CD18, which prevented accumulation of granulocytes but not macrophages, did not lead to major changes in the phenotype, suggesting that skin macrophages but not granulocytes, are important for psoriasis-like dermatitis in this model. Taken together, macrophages are increased in lesional skin of psoriasis, especially in stable plaques, and an increased ratio of M1/M2 macrophages and TNF- $\alpha$ production by macrophages are essential for development of psoriasis (Figure 2).

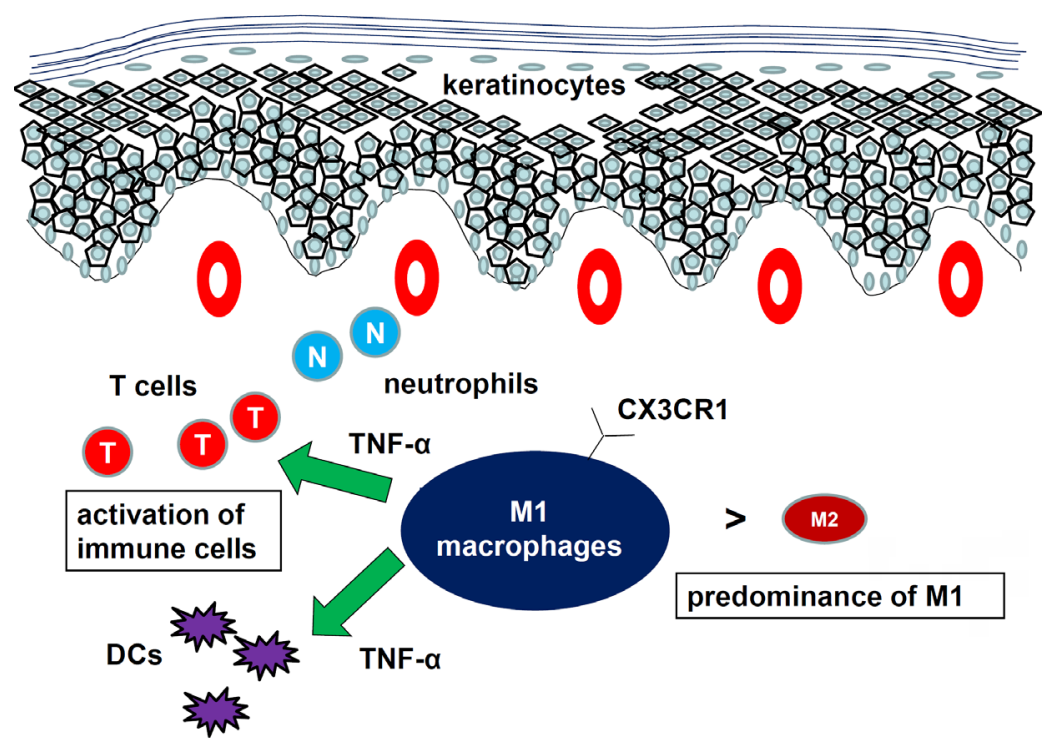

Figure 2. Role of macrophages in psoriasis. The ratio of M1/M2 macrophages is increased. Macrophages produced proinflammatory cytokines to develop psoriasis

\section{Role of macrophages in systemic sclerosis}

Systemic sclerosis (SSc) is a connective tissue disorder characterized by the three main pathological features, including autoimmunity/inflammation, vasculopathy, and fibrosis in multiple organs including skin. Excessive collagen production is mediated by abnormal interactions between endothelial cells, fibroblasts and mononuclear cells, particularly macrophages and Tlymphocytes. CX3CR1 expression levels on peripheral monocytes/macrophages and $\mathrm{T}$ cells were increased in patients with diffuse cutaneous SSc [32]. Moreover, the numbers of CX3CR1-positive cells in the skin and lung were increased in the patients. Endothelial cells in the diseased skin and lung expressed fractalkine, a ligand for CX3CR1, suggesting that recruitment of CX3XR1-expressing monocytes/macrophages into the skin is important for the development of SSc. The importance of macrophages in SSc is also shown by increased numbers of CD163 ${ }^{+}$cells and CD204 ${ }^{+}$cells between the collagen fibers in the diseased skin [33]. In a mouse model using injections of TGF- $\beta$ followed by injections of connective tissue growth factor (CTGF), the overproduction of collagen induced by TGF- $\beta$ was significantly decreased in CCR2-deficient mice, while collagen contents induced by CTGF were restored 
to WT levels [34]. On the other hand, collagen synthesis in CX3CR1-deficient mice decreased by both TGF- $\beta$ and CTGF stimulations, suggesting that both CCR2-expressing macrophages and CX3CR1-expressing macrophages are important in the development of fibrosis. Haploinsufficient loss of Friend leukemia virus integration 1 (Fli1), a potential predisposing factor of SSc, remarkably induced CXCL13 expression in murine macrophages [35]. Serum levels of this chemokine were elevated in SSc patients and correlated positively with skin score and negatively with pulmonary function test results, indicating that CXCL13 expression in macrophages contributes to the development of SSc. In a recent paper, monocyte-derived macrophages, when interferon regulatory factor 8 (IRF8) expression was silenced, displayed an M2 phenotype and expressed profibrotic factors and extracellular matrix (ECM) components [36]. Skin fibrosis was enhanced in myeloid cell-specific IRF8 conditional knockout mice. Thus, migration and activation of macrophages via various chemokines and transcription factors are important for development of SSc.

\section{Role of macrophages in skin malignancy}

It is well-known that tumor-associated macrophages (TAMs), the most frequently found immune cells within the tumor microenvironment, promote tumor growth through promotion of tumor survival pathways and suppression of cytotoxic $\mathrm{T}$ cell responses. In most cases, TAMs show M2 phenotype and they produce various pro-angiogenic cytokines and growth factors such as vascular endothelial growth factor (VEGF), IL-8, and fibroblast growth factor [37-39]. Macrophages can restrict T cell activity through expression of inhibitory immune checkpoint molecules, including PD-L1 and PD-L2, impaired antigen presentation, secretion of immunosuppressive cytokines, and depletion of tryptophan and arginine. The presence of TAMs has been associated with poor prognosis in cancers in various tissues such as bladder, breast, prostate, and colon [40-43]. In malignancy of lymphoid cells, the number of $\mathrm{CD}_{163}{ }^{+}$cells significantly correlated with shorter overall survival in patients with follicular lymphoma, angioimmunoblastic T-cell lymphoma, Hodgkin's lymphoma, and diffuse large B cell lymphoma [44-47].

In case of skin malignancy, elevated numbers of TAMs are often correlated with poor prognosis in melanoma [48]. Expression levels of CD68 in melanoma lesions were dramatically upregulated in the late stage relative to those in the early stage of tumors [49]. Moreover, confocal microscopy studies showed that the percentage of $\mathrm{CD} 206^{+}$cells out of $\mathrm{CD} 68^{+}$cells was much higher at the late stage compared with that at the early stage, suggesting that TAMs were M2 macrophages at advanced melanoma. TAMs enhanced endothelial cell migration and tubule formation and also increased mouse B16 melanoma cell lines [49]. Thus, TAMs enhance angiogenesis and tumor growth in melanoma. TAMs are also important for non-melanoma skin cancers such as cutaneous squamous cell carcinoma (SCC), Merkel cell carcinoma, and extramammary Paget's disease [50-52]. In cutaneous SCC, CD163 ${ }^{+}$TAMs produced protumoral factors and matrix metalloproteinases 9 and 11 [50]. There was co-expression of CD163 and M2 markers, CD209, and CCL18. M1-related genes were also expressed in skin lesions of SCC, suggesting that heterogeneous activation states of TAMs in SCC. Infiltration of immunosuppressive M2 macrophages and regulatory T cells (Tregs) was also seen in Merkel cell carcinoma [51].

Mycosis fungoides (MF) and Sézary syndrome (SS) are the most common types of cutaneous T-cell lymphoma (CTCL). MF is characterized by malignant proliferation of neoplastic T cells in the skin and has a classically prolonged clinical course. Only limited cases progress over years through patch, plaque, and tumor stages, followed by lymph node and visceral involvement [53]. SS is characterized by erythroderma, lymphadenopathy, and leukemic involvement, which means the presence of more than 1,000 per $\mathrm{mm}^{3}$ circulating atypical T-cells with cerebriform nuclei, so-called Sézary cells. Although pathogenesis of CTCL is unknown, a variety of cytokines/chemokines are reported to be involved in the development of the disease $[54,55]$. We demonstrated that the numbers of CD163+ TAMs in lesional skin of CTCL were significantly larger than those in normal skin and that they increased as more tumor cells infiltrated [56]. Moreover, CTCL patients with an increased number of CD163+ TAMs showed worse prognosis.

Cytokines expressed by macrophages are also important in cross talk among skin macrophages, tumor cells, blood vascular endothelial cells, and TILs. CCL18, also called pulmonary and activation-regulated 
chemokine, is induced by alternatively activated macrophages $[57,58]$. TAMs expressed CCL18 in CTCL skin $[59,60]$. It was reported that serum CCL18 levels in patients with CTCL were significantly higher than those of healthy controls and they significantly correlated with skin tumor burden, serum sIL-2R, lactate dehydrogenase, and Th2 cytokines/chemokines levels [60]. Patients with high serum levels of CCL18 showed significantly poor prognosis compared to those with low CCL18 levels. CCL17, also called thymus and activation-regulated chemokine, is also expressed by macrophages in addition to DCs and endothelial cells. This chemokine is a ligand of CCR4, which is expressed by tumor cells in CTCL [61]. It was previously reported that serum CCL17 levels reflect disease activity of CTCL [62].

The role of macrophages in skin malignancy was also examined using mouse models. TAMs derived from mouse B16 melanoma expressed macrophage receptor with collagenous structure, and intravenous administration of its blocking antibodies reprogrammed the TAMs to a proinflammatory phenotype [63]. In another report, IFN- $\beta$ decreased the production of CCL22 from TAMs in mouse B16 melanoma, leading to suppression of tumor growth by the modulation of TIL profiles in vivo [64]. The relationship between macrophages and the progression of T-cell lymphoma was also studied using mouse models $[65,66]$. No tumor formation was seen when MBL2 murine T lymphoma cells was injected into ear skin of C57BL/6. However, induction of skin inflammation by 2,4-dinitrofluorobenzene (DNFB) following MBL2 inoculation lead to progressive high-grade lymphoma [65]. Depletion of macrophages by clodronate-containing liposomes blocked the tumor-promoting effect of DNFB, suggesting the importance of macrophages. The same group also xenografted human CTCL tumor cells in immunocompromised mice and compared tumor development using clodronate-containing liposomes to deplete macrophages in mice [66]. Mice treated with clodronate-containing liposomes showed markedly less tumor growth compared with those treated with phosphate-buffered saline-containing liposomes. Taken together, macrophages have a critical role in the progression skin malignancy through promotion of tumor survival pathways via expression of cytokines and growth factors, interaction with Tregs and myeloid-derived suppressor cells, and suppression of function of TILs by immunosuppressive cytokines and PD-L1 (Figure 3). Selective depletion of TAMs or repolarization of tumor-promoting macrophages to tumor-suppressive macrophages would be a future therapeutic strategy.

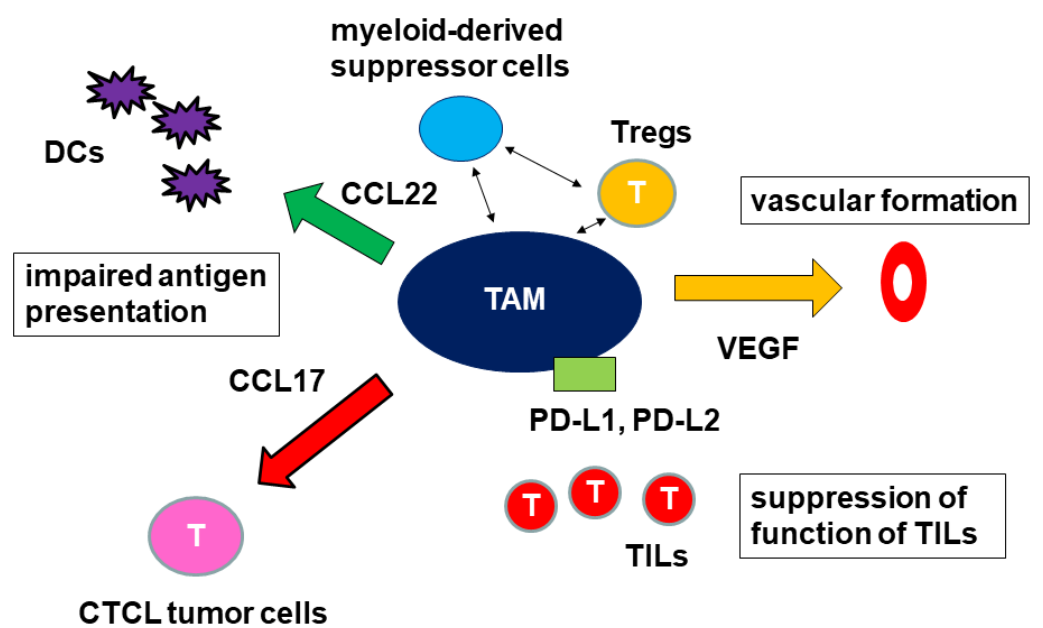

Figure 3. Role of macrophages in skin malignancy. Macrophages interact with Tregs and myeloid-derived suppressor cells, suppressing tumor immunity. Macrophages also mediate vascular formation and chemotaxis of tumor cells

\section{Role of fibroblasts in AD and CHS}

Fibroblasts are spindle-shaped non-migratory cells whose main function is collagen production. It is well known that tissue fibrosis occurs after long-lasting inflammatory status. Therefore, fibroblasts had been thought to terminate immune responses. Now we know that fibroblasts produce many chemokines that can initiate or enhance inflammatory process.

AD is characterized by Th2-dominant immune responses. CCL17 is a representative disease activity maker [67]. CCL17 is produced by dermal fibroblasts stimulated by IL-4. Fibroblasts also produce CCL11 and CCL26, which are eotaxin-1 and eotaxin-3, respectively. These chemokines induce migration of Th2 cells 
and eosinophils. Thymic stromal protein (TSLP) potently activates immature myeloid DCs, which mediates Th2 immune responses by producing CCL17 $[68,69]$. Expression of TSLP, which is strongly expressed by keratinocytes in AD skin, is induced by danger signals such as mechanical injury, pro-inflammatory milieu and proteases. Discovery of periostin has led us to the mechanisms underlying chronicity in AD [70]. Fibroblasts stimulated by Th2 cytokines IL-4 and IL-13 produce periostin, which interacts with $\alpha_{v}$ integrin, a functional periostin receptor on keratinocytes. Stimulated keratinocytes in turn express TSLP, which consequently accelerated Th2-type immune responses. Thus, periostin sets up a vicious circle that links Th2-type immune responses to keratinocyte activation and plays a critical role in the amplification and chronicity of AD. Recently, it was found that chemokine (C-X-C motif) receptor (CXCR) 4 and its ligand CXCL12 were significantly upregulated in $\mathrm{AD}$ [71]. CXCR4+ natural killer T (NKT) cells were enriched both in human AD skin and in AD mouse models. Skin fibroblasts were the main source of the chemokine. Enriched CXCR4 ${ }^{+}$tissue-resident NKT cells/CXCL12 ${ }^{+}$cell cluster may be important for AD. Another recent study using single-cell RNA sequencing on AD skin found a new fibroblast subpopulation expressing COL6A5 and COL18A1, which was unique to lesional AD [72]. These fibroblasts produced CCL2 and CCL19. DCs expressing the CCL19 receptor CCR7 was also unique to $\mathrm{AD}$ lesions, suggesting an important cross talk between fibroblasts and migratory immune cells. Mice whose dermal fibroblasts lacked $I k k 2$ expression spontaneously developed skin inflammation limited to the face [73]. These mice showed phenotypes similar to those of AD patients, including scratching behaviors, which are resistant to immunosuppressive or molecularly targeted drugs. Thus, fibroblasts play important roles in development and maintenance of AD lesions through expression of CCL17, CCL11, CCL26, CXCL12, CCL19, and periostin, interacting with Th2 cells, NKT cells, DCs, and keratinocytes (Figure 4).

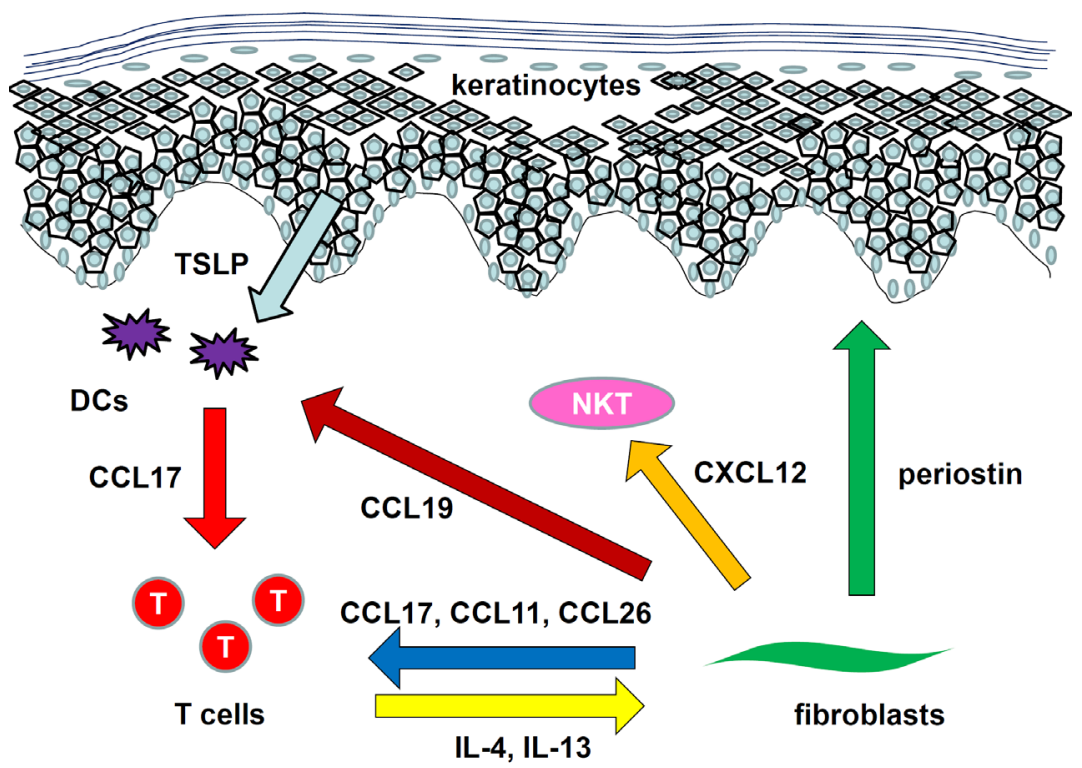

Figure 4. Role of fibroblasts in AD. Fibroblasts express CCL17, CCL11. CCL26, CXCL12, CCL19, and periostin, interacting with T cells, NKT cells, DCs, and keratinocytes

There are only a few studies about the role of fibroblasts in CHS. The importance of fibroblasts has been shown in terms of cytokine expression in CHS [74-77]. CHS is dependent upon the activity of matrix metalloproteinases, which are mainly produced by fibroblasts [74]. CCL2, also called monocyte chemoattractant protein-1, is a potent chemotactic factor for many types of leukocytes including monocytes/macrophages and T cells. This cytokine is mainly expressed by fibroblasts and the expression is increased in CHS. Small interfering RNA (siRNA) for CCL2 significantly suppressed ear swelling in a CHS model [75]. Silencing of signal transducer and activator of transcription 6 (STAT6) by siRNA in dermal fibroblasts inhibited CCL11 production in response to IL-4 and TNF- $\alpha$ in vitro [76]. Local administration of STAT6 siRNA in vivo alleviated CHS responses to chemical haptens, suggesting an important role of STAT6 activation in dermal fibroblasts in Th2-mediated cutaneous diseases. Recruitment of eosinophils, which are the mediator of CHS, into the skin was induced by basophils in cooperation with fibroblasts [77]. Thus, fibroblasts are important in CHS by expressing soluble factors such as CCL2 and CCL11. 


\section{Role of fibroblasts in psoriasis}

Although T cells and DCs are main players in psoriasis, the cross talk between dermal fibroblasts and those migratory immune cells and skin epidermis is also important. Fibroblasts express various cytokines and growth factors such as VEGF, IL-8, a chemoattractant for neutrophils and one of the key cytokines in psoriasis, is expressed by fibroblasts as well as macrophages, endothelial cells, keratinocytes, and neutrophils. Fibroblasts from psoriasis patients secreted more IL-8 than those from normal controls [78]. It is noteworthy that fibroblasts from lesional skin secreted more than those from perilesional skin. Neutrophils released IL-8 and IL-12 and their concentrations got higher when co-cultured with fibroblasts in psoriatic patients. TGF- $\beta$ and keratinocyte growth factor, together with fibronectin and $\alpha 5 \beta 1$ integrin, have been suggested to play a crucial role in the pathogenesis of psoriasis. The expression of $\alpha 5$ integrin, extra domain A-positive fibronectin, keratinocyte growth factor and its receptor fibroblast growth factor receptor 2 was elevated in psoriatic nonlesional skin compared with healthy skin, suggesting that the production of fibronectin by fibroblasts was abnormally regulated even in nonlesional skin from psoriasis patients [79]. With regards to the secretion of cyclooxygenase (COX)-2-derived inflammatory mediators, diminished production of prostaglandin $\mathrm{E}_{2}$ by psoriasis fibroblasts was reported [80]. This phenotype correlated with deficient c-Jun N-terminal kinase activation, in accordance with the hypothesis that alterations in this signaling pathway are associated with psoriasis. Furthermore, conditioned medium from psoriatic fibroblasts promoted the polarization of monocytic cells toward a pro-inflammatory profile, suggesting a prominent role of dermal fibroblasts in the regulation of inflammatory responses through the participation of COX-derived metabolites [80]. This resolutive behavior seems to be defective in psoriatic fibroblasts, offering a possible explanation for the chronic disease.

The presence of oxidative stress such as lipid peroxidation and total antioxidant capacity significantly induced decreased silent information regulator 2 homolog 1 (sirtuin 1) expression level and activity, mitochondrial damage, and apoptosis in psoriatic fibroblasts [81]. Upon sirtuin 1 activation, redox balance was re-established, mitochondrial function was restored, and apoptosis was no longer evident. The changes in the proteomic profile of dermal fibroblasts in the psoriasis lesions was also reported [82]. Dysfunction of fibroblasts in psoriasis arose from the upregulation of proinflammatory factors and antioxidant proteins, as well as those involved in signal transduction and participating in proteolytic processes. Moreover, downregulated proteins in psoriatic fibroblasts were mainly responsible for the transcription/translation processes, glycolysis/ adenosine triphosphate synthesis, and structural molecules [82]. Taken together, fibroblast play important roles in psoriasis, expressing IL-8 and VEGF, production of fibronectin, and changes in the proteomic profiles (Figure 5).

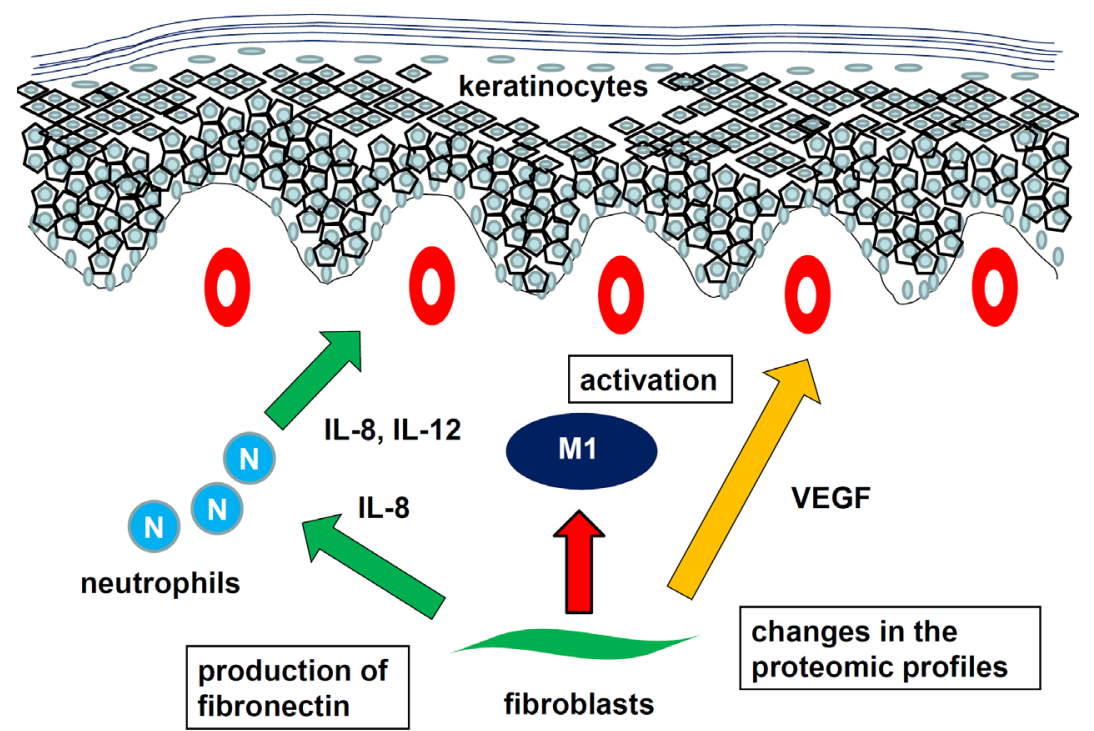

Figure 5. Role of fibroblasts in psoriasis. Fibroblast express IL-8, inducing chemotaxis and activation of neutrophils. Activation of M1 macrophages, production of fibronectin, and changes in the proteomic profiles contribute to development of psoriasis 


\section{Role of fibroblasts in SSc}

Fibrosis in multiple organs is a critical characteristic of SSc. In the involved organs, $\alpha$-smooth muscle actin $(\alpha-S M A)$-positive myofibroblasts produce ECM. The most important factor involved in the activation of SSc dermal fibroblasts is TGF- $\beta$. TGF- $\beta$ expression is prominent around dermal vessels, where there are many migratory immune cells [83]. SSc dermal fibroblasts respond to various stimuli from activated T cells. They are resistant to Th1 cell- and Th2 cell-mediated suppression of collagen production [84]. Dermal fibroblasts from patients with SSc, on the other hand, induce the differentiation of Tregs into Th2-like cells through the IL-33 [85]. Furthermore, SSc dermal fibroblasts suppress the IFN- $\gamma$ expression by T cells through galectin- 9 overproduction, promoting skin fibrosis by establishing the Th2/Th17 dominant microenvironment [86]. Thus, crosstalk between dermal fibroblasts in T cells is essential for development of SSc.

\section{Role of fibroblasts in skin malignancy}

Fibroblasts in tumor microenvironment, called cancer-associated fibroblasts (CAFs), produce ECM and cytokines modulating tumor immunity. CAFs express specific markers such as $\alpha$-SMA. CAF-derived ECM components cause alterations in the ECM composition and initiate the ECM remodeling. Immune regulation of tumors is largely determined by the ECM remodeling because the ECM is responsible for regulating a variety of functions, such as proliferation, differentiation, and morphogenesis. It is well known that CAFs mediate tumor progression in various cancers including skin malignancy. The roles of CAFs in melanoma, especially the cross talk between CAFs and melanoma cells, have been studied. Cellular communication network 2 (CCN2), formerly termed CTGF, was overexpressed by CAFs in melanoma [87, 88]. In human melanoma patients, CCN2 expression negatively correlated with survival and positively correlates with expression of neovascularization markers. Using mouse models, loss of CCN2 in CAFs resulted in reduced CAF activation and reduced tumor-induced neovascularization. CCN2-deficinet CAF expressed reduced levels of $\alpha$-SMA, suggesting an essential role of CCN2 for the differentiation of dermal progenitor cells into a CAF phenotype. TNF receptor associated-factor (TRAF)6, which promotes the malignant phenotype of melanoma cells, was also significantly upregulated in CAFs adjacent to melanoma cells [89]. Functional assays showed that TRAF6 promoted fibroblast proliferation and migration as well as $\alpha$-SMA expression. Moreover, the expression of TRAF6 in fibroblasts promoted the malignant phenotype of melanoma cells in vitro and in vivo. Pigment epithelium-derived factor (PEDF), expressed by fibroblasts, has the opposite effects on tumor cells [90]. While normal dermal fibroblasts expressing high PEDF levels attenuated melanoma growth and angiogenesis in vivo, whereas $P E D F$-depleted fibroblasts promoted tumor growth. Normal fibroblasts in close contact with PEDF-null melanoma cells lost PEDF expression and tumor-suppressive properties. CAFs isolated from melanoma expressed markedly low levels of PEDF. Treatment of patient CAFs and TGF- $\beta$-treated normal fibroblasts with exogenous PEDF decreased the expression of CAF markers and restored PEDF expression. Thus, PEDF maintains tumor-suppressive functions in fibroblasts to prevent CAF conversion.

In addition to the interaction with melanoma cells, there is a cross talk between CAFs and migratory immune cells. CAFs derived from metastatic melanomas sharply interfered with natural killer (NK) cell functions including cytotoxicity and cytokine production in vitro [91]. CAFs inhibited both the IL-2-induced up-regulation of the surface expression of CD336, CD337, and CD226 triggering receptors and the acquisition of cytolytic granules in NK cells. While cell-to-cell contact was required for inducing CD226 modulation, modulation of CD336 and CD337 was due to prostaglandin E2 released by CAFs during coculture. Thus, progression and metastasis of melanoma are mediated by the cross talk among different cell types in the tumor microenvironment and activated CAFs are one of the essential players.

Importance of fibroblasts is also recognized in CTCL [92]. Dermal fibroblasts express CXCL9 and CXCL10 and their receptor CXCR3 is expressed by tumor cells in early MF lesions [93-95]. Interactions between CXCL9, CXCL10, and CXCR3 are regarded important for skin recruitment and accumulation of tumor cells in early stages of MF. In tumor stage MF, tumor cells transform into much larger cells, which tend to express CCR4 rather than CXCR3 $[62,96]$. Circulating tumor cells as well as skin-infiltrating cells in SS also express CCR4. CCL17, a ligand of CCR4 and CCR8, serves to recruit cells expressing these chemokine receptors. CCL17 is 
expressed by fibroblasts in lesional skin of MF and SS, suggesting important roles of CCL17-CCR4 interaction in preferential traffic of tumor cells of MF and SS to the skin. In addition to CCR4 and CCR10, tumor cells of SS express CXCR4, which is a receptor for CXCL12 [97]. CXCL12 is also produced by fibroblasts, especially CAFs. CXCL12/CXCR4 axis reduced the sensitivity of CTCL cell lines to doxorubicin, and enhanced their migration when co-cultured with fibroblasts [98].

CCL26 and CCL11 induces chemotaxis of CCR3-expressing cells such and eosinophils and subpopulations of Th2 cells. We found that CCL26 and CCL11 expression by dermal fibroblasts is increased in lesional skin of advanced CTCL [99]. Migration of Th2 cells into the lesional skin promotes a Th2-dominant microenvironment. A recent study revealed that MF skin fibroblasts showed increased expression of CAF markers with increasing stage compared to normal fibroblasts [100]. CTCL cell lines cultured with MF fibroblasts retained high expression of MF biomarkers. Taken together, fibroblasts have a critical role in the progression skin malignancy via expression of cytokines such as CCN2, TRAF6, CXCL12, and suppression NK functions, and establishment of Th2-dominant microenvironment (Figure 6).

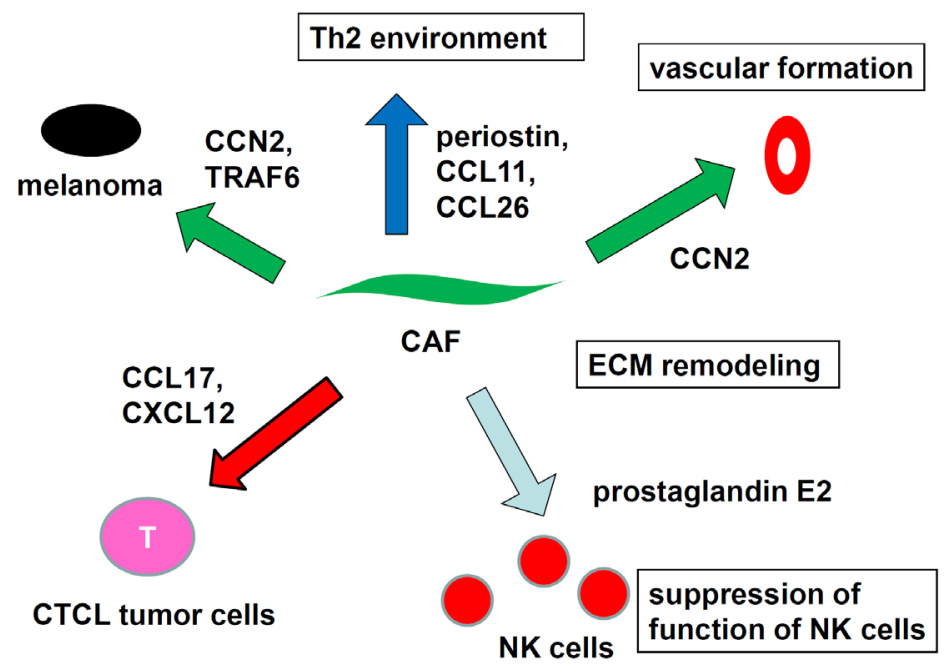

Figure 6. Role of fibroblasts in skin malignancy. Fibroblasts express cytokines, inducing progression of skin malignancy Suppression NK functions and establishment of Th2-dominant microenvironment also contribute to the pathogenesis

\section{Conclusions}

In this review, various kinds of cross talk among macrophages, fibroblasts, and migratory immune cells such as T cells, DCs, and NK cells in skin inflammatory diseases such as AD and psoriasis is summarized. Macrophages and fibroblasts also have interactions with tumor cells in melanoma and CTCL, promoting tumor progression and metastasis. Those skin-resident cells have been neglected for a long time, but they are attracting therapeutic targets in the near future.

\section{Abbreviations}

AD: atopic dermatitis

$\alpha$-SMA: $\alpha$-smooth muscle actin

CAFs: cancer-associated fibroblasts

CCL: CC chemokine ligand

CCN2: cellular communication network 2

CCR: CC chemokine receptor

CHS: contact hypersensitivity

CTCL: cutaneous T-cell lymphoma

CTGF: connective tissue growth factor

CXCL: C-X-C motif chemokine ligand 
CXCR: chemokine (C-X-C motif) receptor

CX3CR1: CX3C chemokine receptor 1

DCs: dendritic cells

ECM: extracellular matrix

IL: interleukin

IMQ: imiquimod

MF: mycosis fungoides

NK: natural killer

NKT: natural killer $\mathrm{T}$

PD-L: programmed death-ligand

PEDF: pigment epithelium-derived factor

SCC: squamous cell carcinoma

SS: Sézary syndrome

SSc: systemic sclerosis

TAMs: tumor-associated macrophages

TGF: transforming growth factor

Th: T helper

TILs: tumor-infiltrating T cell

TNF: tumor necrosis factor

TRAF: TNF receptor associated-factor

Tregs: regulatory $\mathrm{T}$ cells

VEGF: vascular endothelial growth factor

WT: wild type

\section{Declarations}

Author contributions

The author contributed solely to the work.

\section{Conflicts of interest}

The author declares that there are no conflicts of interest.

\section{Ethical approval}

Not applicable.

\section{Consent to participate}

Not applicable.

Consent to publication

Not applicable.

\section{Availability of data and materials}

Not applicable.

\section{Funding}

Not applicable.

\section{Copyright}

(c) The Author(s) 2021. 


\section{References}

1. Ho AW, Kupper TS. T cells and the skin: from protective immunity to inflammatory skin disorders. Nat Rev Immunol. 2019;19:490-502.

2. Cheuk S, Wikén M, Blomqvist L, Nylén S, Talme T, Ståhle M, et al. Epidermal Th22 and Tc17 cells form a localized disease memory in clinically healed psoriasis. J Immunol. 2014;192:3111-20.

3. Nghiem PT, Bhatia S, Lipson EJ, Kudchadkar RR, Miller NJ, Annamalai L, et al. PD-1 blockade with pembrolizumab in advanced Merkel-cell carcinoma. N Engl J Med. 2016;374:2542-52.

4. Schadendorf D, van Akkooi ACJ, Berking C, Griewank KG, Gutzmer R, Hauschild A, et al. Melanoma. Lancet. 2018;392:971-84.

5. Harms PW, Harms KL, Moore PS, DeCaprio JA, Nghiem P, Wong MKK, et al. The biology and treatment of Merkel cell carcinoma: current understanding and research priorities. Nat Rev Clin Oncol. 2018;15:763-76.

6. Biswas SK, Mantovani A. Macrophage plasticity and interaction with lymphocyte subsets: cancer as a paradigm. Nat Immunol. 2010;11:889-96.

7. Gordon S, Martinez FO. Alternative activation of macrophages: mechanism and functions. Immunity. 2010;32:593-604.

8. Martinez FO, Gordon S, Locati M, Mantovani A. Transcriptional profiling of the human monocyte-tomacrophage differentiation and polarization: new molecules and patterns of gene expression. J Immunol. 2006;177:7303-11.

9. Mantovani A, Sica A, Sozzani S, Allavena P, Vecchi A, Locati M. The chemokine system in diverse forms of macrophage activation and polarization. Trends Immunol. 2004;25:677-86.

10. Mosser DM. The many faces of macrophage activation. J Leukoc Biol. 2003;73:209-12.

11. Goerdt $\mathrm{S}$, Orfanos CE. Other functions, other genes: alternative activation of antigen-presenting cells. Immunity. 1999;10:137-42.

12. Saeki H, Nakahara T, Tanaka A, Kabashima K, Sugaya M, Murota H, et al. Clinical practice guidelines for the management of atopic dermatitis 2016. J Dermatol. 2016;43:1117-45.

13. Xu X, van Galen LS, Koh MJA, Bajpai R, Thng S, Yew YW, et al. Factors influencing quality of life in children with atopic dermatitis and their caregivers: a cross-sectional study. Sci Rep. 2019;9:15990.

14. Ražnatović Đurović M, Janković J, Tomić Spirić V, Relić M, Sojević Timotijević Z, Ćirković A, et al. Does age influence the quality of life in children with atopic dermatitis? PLoS One. 2019;14:e0224618.

15. Kabashima K. New concept of the pathogenesis of atopic dermatitis: interplay among the barrier, allergy, and pruritus as a trinity. J Dermatol Sci. 2013;70:3-11.

16. Salimi M, Barlow JL, Saunders SP, Xue L, Gutowska-Owsiak D, Wang X, et al. A role for IL-25 and IL-33driven type-2 innate lymphoid cells in atopic dermatitis. J Exp Med. 2013;210:2939-50.

17. Imai Y, Yasuda K, Sakaguchi Y, Haneda T, Mizutani H, Yoshimoto T, et al. Skin-specific expression of IL-33 activates group 2 innate lymphoid cells and elicits atopic dermatitis-like inflammation in mice. Proc Natl Acad Sci USA. 2013;110:13921-6.

18. van de Veen W, Akdis M. The use of biologics for immune modulation in allergic disease. J Clin Invest. 2019;130:1452-62.

19. Kiekens RC, Thepen T, Oosting AJ, Bihari IC, Van De Winkel JG, Bruijnzeel-Koomen CA, et al. Heterogeneity within tissue-specific macrophage and dendritic cell populations during cutaneous inflammation in atopic dermatitis. Br J Dermatol. 2001;145:957-65.

20. Suwanpradid J, Shih M, Pontius L, Yang B, Birukova A, Guttman-Yassky E, et al. Arginase1 deficiency in monocytes/macrophages upregulates inducible nitric oxide synthase to promote cutaneous contact hypersensitivity. J Immunol. 2017;199:1827-34. 
21. Otobe S, Hisamoto T, Miyagaki T, Morimura S, Suga H, Sugaya M, et al. CX3CR1 deficiency attenuates DNFBinduced contact hypersensitivity through skewed polarization towards M2 phenotype in macrophages. Int J Mol Sci. 2020;21:7401.

22. Natsuaki Y, Egawa G, Nakamizo S, Ono S, Hanakawa S, Okada T, et al. Perivascular leukocyte clusters are essential for efficient activation of effector T cells in the skin. Nat Immunol. 2014;15:1064-9.

23. Tuckermann JP, Kleiman A, Moriggl R, Spanbroek R, Neumann A, Illing A, et al. Macrophages and neutrophils are the targets for immune suppression by glucocorticoids in contact allergy. J Clin Invest. 2007;117:1381-90.

24. Griffiths CEM, Armstrong AW, Gudjonsson JE, Barker JNWN. Psoriasis. Lancet. 2021;397:1301-15.

25. Ghoreschi K, Balato A, Enerbäck C, Sabat R. Therapeutics targeting the IL-23 and IL-17 pathway in psoriasis. Lancet. 2021;397:754-66.

26. Lu CH, Lai CY, Yeh DW, Liu YL, Su YW, Hsu LC, et al. Involvement of M1 macrophage polarization in endosomal toll-like receptors activated psoriatic inflammation. Mediators Inflamm. 2018;2018:3523642.

27. Terhorst D, Chelbi R, Wohn C, Malosse C, Tamoutounour S, Jorquera A, et al. Dynamics and transcriptomics of skin dendritic cells and macrophages in an imiquimod-induced, biphasic mouse model of psoriasis. J Immunol. 2015;195:4953-61.

28. Morimura $S$, Oka T, Sugaya $M$, Sato S. CX3CR1 deficiency attenuates imiquimod-induced psoriasis-like skin inflammation with decreased M1 macrophages. J Dermatol Sci. 2016;82:175-88.

29. Combadiere C, Potteaux S, Gao JL, Esposito B, Casanova S, Lee EJ, et al. Decreased atherosclerotic lesion formation in CX3CR1/apolipoprotein E double knockout mice. Circulation. 2003;107:1009-16.

30. Wang H, Peters T, Kess D, Sindrilaru A, Oreshkova T, Van Rooijen N, et al. Activated macrophages are essential in a murine model for $\mathrm{T}$ cell-mediated chronic psoriasiform skin inflammation. J Clin Invest. 2006;116:2105-14.

31. Stratis A, Pasparakis M, Rupec RA, Markur D, Hartmann K, Scharffetter-Kochanek K, et al. Pathogenic role for skin macrophages in a mouse model of keratinocyte-induced psoriasis-like skin inflammation. J Clin Invest. 2006;116:2094-104.

32. Hasegawa M, Sato S, Echigo T, Hamaguchi Y, Yasui M, Takehara K. Up regulated expression of fractalkine/ CX3CL1 and CX3CR1 in patients with systemic sclerosis. Ann Rheum Dis. 2005;64:21-8.

33. Higashi-Kuwata N, Jinnin M, Makino T, Fukushima S, Inoue Y, Muchemwa FC, et al. Characterization of monocyte/macrophage subsets in the skin and peripheral blood derived from patients with systemic sclerosis. Arthritis Res Ther. 2010;12:R128.

34. Arai M, Ikawa Y, Chujo S, Hamaguchi Y, Ishida W, Shirasaki F, et al. Chemokine receptors CCR2 and CX3CR1 regulate skin fibrosis in the mouse model of cytokine-induced systemic sclerosis. J Dermatol Sci. 2013;69:250-8.

35. Taniguchi T, Miyagawa T, Toyama S, Yamashita T, Nakamura K, Saigusa R, et al. CXCL13 produced by macrophages due to Fli1 deficiency may contribute to the development of tissue fibrosis, vasculopathy and immune activation in systemic sclerosis. Exp Dermatol. 2018;27:1030-7.

36. Ototake Y, Yamaguchi Y, Asami M, Komitsu N, Akita A, Watanabe T, et al. Downregulated IRF8 in monocytes and macrophages of patients with systemic sclerosis may aggravate the fibrotic phenotype. J Invest Dermatol. 2021;141:1954-63.

37. Qian BZ, Pollard JW. Macrophage diversity enhances tumor progression and metastasis. Cell. 2010;141:39-51.

38. Mantovani A, Allavena P, Sica A, Balkwill F. Cancer related inflammation. Nature. 2008;454:436-44.

39. Pollard JW. Tumour-educated macrophages promote tumour progression and metastasis. Nat Rev Cancer. 2004;4:71-8. 
40. Flamm J, Benesch A. Tumor-associated mononuclear cell infiltrate and recurrence rate in the superficial urothelial carcinoma of the urinary bladder. Urol Int. 1986;41:187-91.

41. Leek RD, Landers RJ, Harris AL, Lewis CE. Necrosis correlates with high vascular density and focal macrophage infiltration in invasive carcinoma of the breast. Br J Cancer. 1999;79:991-5.

42. Shimura S, Yang G, Ebara S, Wheeler TM, Frolov A, Thompson TC. Reduced infiltration of tumorassociated macrophages in human prostate cancer: association with cancer progression. Cancer Res. 2000;60:5857-61.

43. Forssell J, Oberg A, Henriksson ML, Stenling R, Jung A, Palmqvist R. High macrophage infiltration along the tumor front correlates with improved survival in colon cancer. Clin Cancer Res. 2007;13:1472-9.

44. Clear AJ, Lee AM, Calaminici M, Ramsay AG, Morris KJ, Hallam S, et al. Increased angiogenic sprouting in poor prognosis FL is associated with elevated numbers of $\mathrm{CD} 163^{+}$macrophages within the immediate sprouting microenvironment. Blood. 2010;115:5053-6.

45. Niino D, Komohara Y, Murayama T, Aoki R, Kimura Y, Hashikawa K, et al. Ratio of M2 macrophage expression is closely associated with poor prognosis for Angioimmunoblastic T-cell lymphoma (AITL). Pathol Int. 2010;60:278-83.

46. Zaki MA, Wada N, Ikeda J, Shibayama H, Hashimoto K, Yamagami T, et al. Prognostic implication of types of tumor-associated macrophages in Hodgkin lymphoma. Virchows Arch. 2011;459:361-6.

47. Wada N, Zaki MA, Hori Y, Hashimoto K, Tsukaguchi M, Tatsumi Y, et al. Tumour-associated macrophages in diffuse large B-cell lymphoma: a study of the Osaka Lymphoma Study Group. Histopathology. 2012;60:313-9.

48. Makitie T, Summanen P, Tarkkanen A, Kivela T. Tumor-infiltrating macrophages (CD68 ${ }^{+}$cells) and prognosis in malignant uveal melanoma. Invest Ophthalmol Vis Sci. 2001;42:1414-21.

49. Chen P, Huang Y, Bong R, Ding Y, Song N, Wang X, et al. Tumor-associated macrophages promote angiogenesis and melanoma growth via adrenomedullin in a paracrine and autocrine manner. Clin Cancer Res. 2011;17:7230-9.

50. Pettersen JS, Fuentes-Duculan J, Suárez-Fariñas M, Pierson KC, Pitts-Kiefer A, Fan L, et al. Tumorassociated macrophages in the cutaneous SCC microenvironment are heterogeneously activated. J Invest Dermatol. 2011;131:1322-30.

51. Gaiser MR, Weis CA, Gaiser T, Jiang H, Buder-Bakhaya K, Herpel E, et al. Merkel cell carcinoma expresses the immunoregulatory ligand CD200 and induces immunosuppressive macrophages and regulatory $\mathrm{T}$ cells. Oncoimmunology. 2018;7:1-7.

52. Kambayashi Y, Fujimura T, Furudate S, Asano M, Kakizaki A, Aiba S. The possible interaction between receptor activator of nuclear factor kappa-B ligand (RANKL) expressed by extramammary Paget cells and its ligand on dermal macrophages. J Invest Dermatol. 2015;135:2547-50.

53. Hwang ST, Janik JE, Jaffe ES, Wilson WH. Mycosis fungoides and Sézary syndrome. Lancet. 2008;371:945-57.

54. Wu XS, Lonsdorf AS, Hwang ST. Cutaneous T-cell lymphoma: roles for chemokines and chemokine receptors. J Invest Dermatol. 2009;129:1115-9.

55. Sugaya M. Chemokines and cutaneous lymphoma. J Dermatol Sci. 2010;59:81-9.

56. Sugaya M, Miyagaki T, Ohmatsu H, Suga H, Kai H, Kamata M, et al. Association of the numbers of CD163+ cells in lesional skin and serum levels of soluble CD163 with disease progression of cutaneous $\mathrm{T}$ cell lymphoma. J Dermatol Sci. 2012;68:45-51.

57. Kodelja V, Muller C, Politz O, Hakij N, Orfanos CE, Goerdt S. Alternative macrophage activation-associated CC-chemokine-1, a novel structural homologue of macrophage inflammatory protein-1 alpha with a Th2-associated expression pattern. J Immunol. 1998;160:1411-8.

58. Song E, Ouyang N, Hörbelt M, Antus B, Wang M, Exton MS. Influence of alternatively and classically activated macrophages on fibrogenic activities of human fibroblasts. Cell Immunol. 2000;204:19-28. 
59. Günther C, Zimmermann N, Berndt N, Grosser M, Stein A, Koch A, et al. Up-regulation of the chemokine CCL18 by macrophages is a potential immunomodulatory pathway in cutaneous T-cell lymphoma. Am J Pathol. 2011;179:1434-42.

60. Miyagaki T, Sugaya M, Suga H, Ohmatsu H, Fujita H, Asano Y, et al. Increased CCL18 expression in patients with cutaneous T-cell lymphoma: association with disease severity and prognosis. J Eur Acad Dermatol Venereol. 2013;27:e60-7.

61. Sugaya M, Morimura S, Suga H, Kawaguchi M, Miyagaki T, Ohmatsu H, et al.CCR4 is expressed on infiltrating cells in lesional skin of early mycosis fungoides and atopic dermatitis. J Dermatol. 2015;42:613-5.

62. Kakinuma T, Sugaya M, Nakamura K, Kaneko F, Wakugawa M, Matsushima K, et al. Thymus and activationregulated chemokine (TARC/CCL17) in mycosis fungoides: serum TARC levels reflect the disease activity of mycosis fungoides. J Am Acad Dermatol. 2003;48:23-30.

63. Georgoudaki AM, Prokopec KE, Boura VF, Hellqvist E, Sohn S, Östling J, et al. Reprogramming tumorassociated macrophages by antibody targeting inhibits cancer progression and metastasis. Cell Rep. 2016;15:2000-11.

64. Kakizaki A, Fujimura T, Furudate S, Kambayashi Y, Yamauchi T, Yagita H, et al. Immunomodulatory effect of peritumoral administration of interferon-beta on melanoma through tumor-associated macrophages. Oncoimmunology. 2015;4:1-10.

65. Wu X, Sells RE, Hwang ST. Upregulation of inflammatory cytokines and oncogenic signal pathways preceding tumor formation in a murine model of T-cell lymphoma in skin. J Invest Dermatol. 2011;131:1727-34.

66. Wu X, Schulte BC, Zhou Y, Haribhai D, Mackinnon AC, Plaza JA, et al. Depletion of M2-like tumorassociated macrophages delays cutaneous T-cell lymphoma development in vivo. J Invest Dermatol. 2014;134:2814-22.

67. Kakinuma T, Nakamura K, Wakugawa M, Mitsui H, Tada Y, Saeki H, et al. Thymus and activation-regulated chemokine in atopic dermatitis: serum thymus and activation-regulated chemokine level is closely related with disease activity. J Allergy Clin Immunol. 2001;107:535-41.

68. Soumelis V, Reche PA, Kanzler H, Yuan W, Edward G, Homey B, et al. Human epithelial cells trigger dendritic cell mediated allergic inflammation by producing TSLP. Nat Immunol. 2002;3:673-80.

69. Ebner S, Nguyen VA, Forstner M, Wang YH, Wolfram D, Liu YJ, et al. Thymic stromal lymphopoietin converts human epidermal Langerhans cells into antigen-presenting cells that induce proallergic $\mathrm{T}$ cells. J Allergy Clin Immunol. 2007;119:982-90.

70. Masuoka M, Shiraishi H, Ohta S, Suzuki S, Arima K, Aoki S, et al. Periostin promotes chronic allergic inflammation in response to Th2 cytokines. J Clin Invest. 2012;122:2590-600.

71. Sun Z, Kim JH, Kim SH, Kim HR, Zhang K, Pan Y, et al. Skin-resident natural killer T cells participate in cutaneous allergic inflammation in atopic dermatitis. J Allergy Clin Immunol. 2021;147:1764-77.

72. He H, Suryawanshi H, Morozov P, Gay-Mimbrera J, Del Duca E, Kim HJ, e al. Single-cell transcriptome analysis of human skin identifies novel fibroblast subpopulation and enrichment of immune subsets in atopic dermatitis. J Allergy Clin Immunol. 2020;145:1615-28.

73. Nunomura S, Ejiri N, Kitajima M, Nanri Y, Arima K, Mitamura Y, et al. Establishment of a mouse model of atopic dermatitis by deleting Ikk2 in dermal fibroblasts. J Invest Dermatol. 2019;139:1274-83.

74. Pilcher BK, Wang M, Qin XJ, Parks WC, Senior RM, Welgus HG. Role of matrix metalloproteinases and their inhibition in cutaneous wound healing and allergic contact hypersensitivity. Ann N Y Acad Sci. 1999;878:12-24.

75. Ishimoto T, Takei Y, Yuzawa Y, Hanai K, Nagahara S, Tarumi Y, et al. Downregulation of monocyte chemoattractant protein-1 involving short interfering RNA attenuates hapten-induced contact hypersensitivity. Mol Ther. 2008;16:387-95. 
76. Hosoya K, Satoh T, Yamamoto Y, Saeki K, Igawa K, Okano M, et al. Gene silencing of STAT6 with siRNA ameliorates contact hypersensitivity and allergic rhinitis. Allergy. 2011;66:124-31.

77. Nakashima C, Otsuka A, Kitoh A, Honda T, Egawa G, Nakajima S, et al. Basophils regulate the recruitment of eosinophils in a murine model of irritant contact dermatitis. J Allergy Clin Immunol. 2014;134:100-7.

78. Glowacka E, Lewkowicz P, Rotsztejn H, Zalewska A. IL-8, IL-12 and IL-10 cytokines generation by neutrophils, fibroblasts and neutrophils- fibroblasts interaction in psoriasis. Adv Med Sci. 2010;55:254-60.

79. Gubán B, Vas K, Balog Z, Manczinger M, Bebes A, Groma G, et al. Abnormal regulation of fibronectin production by fibroblasts in psoriasis. Br J Dermatol. 2016;174:533-41.

80. Arasa J, Terencio MC, Andrés RM, Marín-Castejón A, Valcuende-Cavero F, Payá M, et al. Defective induction of COX-2 expression by psoriatic fibroblasts promotes pro-inflammatory activation of macrophages. Front Immunol. 2019;10:536.

81. Becatti M, Barygina V, Mannucci A, Emmi G, Prisco D, Lotti T, et al. Sirt1 protects against oxidative stressinduced apoptosis in fibroblasts from psoriatic patients: A new insight into the pathogenetic mechanisms of psoriasis. Int J Mol Sci. 2018;19:1572.

82. Gęgotek A, Domingues P, Wroński A, Skrzydlewska E. Changes in proteome of fibroblasts isolated from psoriatic skin lesions. Int J Mol Sci. 2020;21:5363.

83. Kulozik M, Hogg A, Lankat-Buttgereit B, Krieg T. Co-localization of transforming growth factor beta 2 with alpha 1(I) procollagen mRNA in tissue sections of patients with systemic sclerosis. J Clin Invest. 1990;86:917-22.

84. Chizzolini C, Parel Y, De Luca C, Tyndall A, Akesson A, Scheja A, et al. Systemic sclerosis Th2 cells inhibit collagen production by dermal fibroblasts via membrane-associated tumor necrosis factor alpha. Arthritis Rheum. 2003;48:2593-604.

85. MacDonald KG, Dawson NA, Huang Q Dunne JV, Levings MK, Broady R. Regulatory T cells produce profibrotic cytokines in the skin of patients with systemic sclerosis. J Allergy Clin Immunol. 2015;135:e946-9

86. Saigusa R, Asano Y, Nakamura K, Hirabayashi M, Miura S, Yamashita T, et al. Systemic sclerosis dermal fibroblasts suppress Th1 cytokine production via galectin-9 overproduction due to Fli1 deficiency. J Invest Dermatol. 2017;137:1850-9.

87. Hutchenreuther J, Vincent K, Norley C, Racanelli M, Gruber SB, Johnson TM, et al. Activation of cancerassociated fibroblasts is required for tumor neovascularization in a murine model of melanoma. Matrix Biol. 2018;74:52-61.

88. Tsang M, Quesnel K, Vincent K, Hutchenreuther J, Postovit LM, Leask A. Insights into fibroblast plasticity: cellular communication network 2 is required for activation of cancer-associated fibroblasts in a murine model of melanoma. Am J Pathol. 2020;190:206-21.

89. Guo Y, Zhang X, Zeng W, Zhang J, Cai L, Wu Z, et al. TRAF6 activates fibroblasts to cancer-associated fibroblasts through FGF19 in tumor microenvironment to benefit the malignant phenotype of melanoma cells. J Invest Dermatol. 2020;140:2268-79.

90. Nwani NG, Deguiz ML, Jimenez B, Vinokour E, Dubrovskyi O, Ugolkov A, et al. Melanoma cells block PEDF production in fibroblasts to induce the tumor-promoting phenotype of cancer-associated fibroblasts. Cancer Res. 2016;76:2265-76.

91. Balsamo M, Scordamaglia F, Pietra G, Manzini C, Cantoni C, Boitano M, et al. Melanoma-associated fibroblasts modulate NK cell phenotype and antitumor cytotoxicity. Proc Natl Acad Sci U S A. 2009;106:20847-52.

92. Miyagaki T, Sugaya M. Immunological milieu in mycosis fungoides and Sézary syndrome. J Dermatol. 2014;41:11-8. 
93. Sarris AH, Esgleyes-Ribot T, Crow M, Broxmeyer HE, Karasavvas N, Pugh W, et al. Cytokine loops involving interferon- $\gamma$ and IP-10, a cytokine chemotactic for CD4+ lymphocytes: an explanation for the epidermotropism of cutaneous T-cell lymphoma? Blood. 1995;86:651-8.

94. Lu D, Duvic M, Medeiros LJ, Luthra R, Dorfman DM, Jones D. The T-cell chemokine receptor CXCR3 is expressed highly in low-grade mycosis fungoides. Am J Clin Pathol. 2001;115:413-21.

95. Kallinich T, Muche JM, Qin S, Sterry W, Audring H, Kroczek RA. Chemokine receptor expression on neoplastic and reactive $\mathrm{T}$ cells in the skin at different stages of mycosis fungoides. J Invest Dermatol. 2003;121:1045-52.

96. Ferenczi K, Fuhlbrigge RC, Pinkus J, Pinkus GS, Kupper TS. Increased CCR4 expression in cutaneous T cell lymphoma. J Invest Dermatol. 2002;119:1405-10.

97. Narducci MG, Scala E, Bresin A, Caprini E, Picchio MC, Remotti D, et al. Skin homing of Sezary cells involves SDF-1-CXCR4 signaling and down-regulation of CD26/dipeptidylpeptidase IV. Blood. 2006;107:1108-15.

98. Aronovich A, Moyal L, Gorovitz B, Amitay-Laish I, Naveh HP, Forer Y, et al. Cancer-associated fibroblasts in mycosis fungoides promote tumor cell migration and drug resistance through CXCL12/CXCR4. J Invest Dermatol. 2021;141:619-27.

99. Miyagaki T, Sugaya M, Fujita H, Ohmatsu H, Kakinuma T, Kadono T, et al. Eotaxins and CCR3 interaction regulates the Th2 environment of cutaneous T-cell lymphoma. J Invest Dermatol. 2010;130:2304-11.

100. Mehdi SJ, Moerman-Herzog A, Wong HK. Normal and cancer fibroblasts differentially regulate TWIST1, TOX and cytokine gene expression in cutaneous T-cell lymphoma. BMC Cancer. 2021;21:492. 